\title{
Higher Order Crack Tip Fields for Physical Weak-Discontinuous Crack of FGMs Cylindrical Shell with Reissner's Effect
}

\author{
Xiao Chong ${ }^{1, a}$, Yao Dai ${ }^{2, b}$, Yumeng Shi, ${ }^{3, b}$ and Jingwen Pan ${ }^{4, b}$
}

${ }^{1}$ Changping School of Noncommissioned Officer, Academy of Equipment, Beijing, 101416, China

${ }^{2,4}$ Academy of Armored Force Engineering, Beijing, 100072, China

${ }^{3}$ China Academy of Launch Vehicle Technology

achongxiao2005@163.com, ${ }^{\text {b }}$ dai_yao@sina.com

Keywords: crack tip fields, FGMs, Reissner's effect, cylindrical shell

\begin{abstract}
The higher order crack tip fields for physical weak-discontinuous problem of cylindrical shells are investigated. The crack located at the interface between homogeneous materials and exponential functionally graded materials. The governing equations are derived for homogeneous materials and FGMs regions considering transverse shear deformation effect. The higher order crack tip fields of two regions are obtained by the eigen-expansion method, respectively. Finally, the whole crack tip high order fields are assembled and given.
\end{abstract}

\section{Introduction}

Shell-like structural components comprise major load-carrying components in aircraft and space vehicles such as aircraft fuselage, reusable launch vehicles, solid propellant rocket motors, and spacecraft use propellant tanks [1]. Actually, the inner crack in a shell structure always leads to its failure, so the security assessment in engineering has attracted the great public attention. Before, Kirchhoff's theory is mainly adopted in fracture studies of shells, including various spherical shell, cylindrical shell and so on [2]. However, this theory will lead to several problems like the difference of stress singularity, and the difference of stress argument. Therefore, Reissner's theory is widely adopted which considered the effect of shearing deformation, and meanwhile, turned the basic equations into ten-order partial differential equations. In order to consider the above character in the shell structure, a lot of studies have been done [3]. In the engineering applications, FGMs mainly appear in the coating and interface layer, forming lots of interface structures. Due to the feature of production technology, there are a number of interface defects in structures. Furthermore, due to differences in material performance across interfaces. In this paper, the physical weak-discontinuous problem of an interfacial crack is considered. As shown in Fig.1, the area below the interface is homogeneous materials region, and the one above the interface is FGMs region. The gradient direction is along y-axis. In this paper, we extend the Williams' solution to physical weak-discontinuous problem of an interfacial crack between homogeneous material and FGMs cylindrical shells and the higher order fields are obtained.

\section{The Basic Equations}

The elastic modulus function form of FGMs is assumed to be

$$
E^{(2)}=E^{(1)} e^{\beta y}=E^{(1)} e^{\beta r \sin \theta}
$$

where, $E^{(1)}$ is the elastic modulus of homogeneous material, and $\beta \geq 0$ are the non-homogeneity parameter. The variation of Poisson's ratios has very insignificant effect on the stress intensity factor of non-homogeneous materials[4]. So, Poisson's ratio is assumed to be the constant $\mu$. 


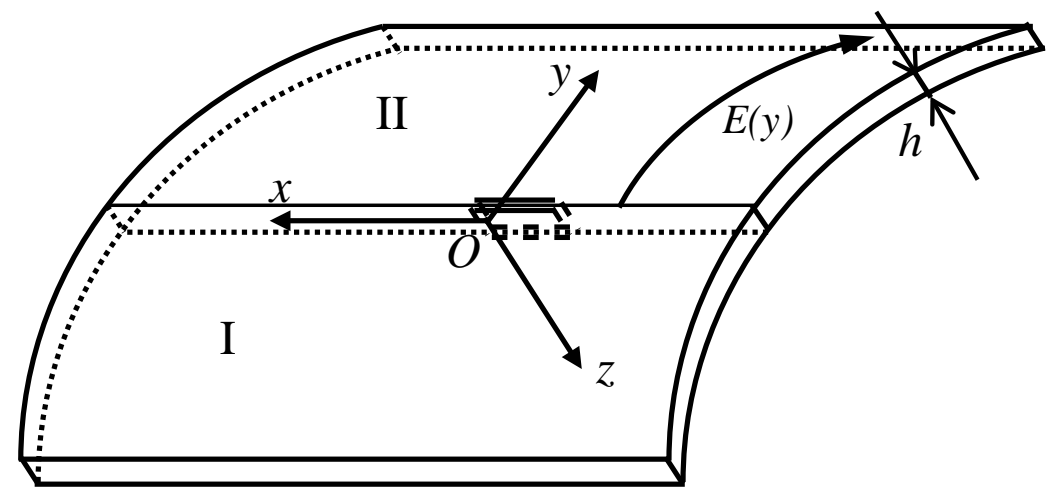

Fig.1 The interfacial crack

Assumed the transverse loading of the cylindrical shell is to be zero, the governing equations for the homogenous material cylindrical shell with Reissner's effect are

$$
\left\{\begin{array}{c}
D^{(k)}\left[\frac{\partial^{2} \varphi_{x}^{(k)}}{\partial x^{2}}+\frac{1-\mu}{2} \frac{\partial^{2} \varphi_{x}^{(k)}}{\partial y^{2}}+\frac{1+\mu}{2} \frac{\partial^{2} \varphi_{y}^{(k)}}{\partial x \partial y}\right)+C^{(k)}\left(\frac{\partial w^{(k)}}{\partial x}-\varphi_{x}^{(k)}\right)+\frac{h^{3}}{24(1+\mu)} \frac{\partial E^{(k)}}{\partial y}\left(\frac{\partial \varphi_{x}^{(k)}}{\partial y}+\frac{\partial \varphi_{y}^{(k)}}{\partial x}\right)=0 \\
D^{(k)}\left[\frac{\partial^{2} \varphi_{y}^{(k)}}{\partial y^{2}}+\frac{1-\mu}{2} \frac{\partial^{2} \varphi_{y}^{(k)}}{\partial x^{2}}+\frac{1+\mu}{2} \frac{\partial^{2} \varphi_{x}^{(k)}}{\partial x \partial y}\right)+C^{(k)}\left(\frac{\partial w^{(k)}}{\partial y}-\varphi_{y}^{(k)}\right)+\frac{h^{3}}{12\left(1-\mu^{2}\right)} \frac{\partial E^{(k)}}{\partial y}\left(\frac{\partial \varphi_{y}^{(k)}}{\partial y}+\mu \frac{\partial \varphi_{x}^{(k)}}{\partial x}\right)=0 \\
C^{(k)}\left(\nabla^{2} w^{(k)}-\frac{\partial \varphi_{x}^{(k)}}{\partial x}-\frac{\partial \varphi_{y}^{(k)}}{\partial y}\right)+\kappa \frac{\partial^{2} \psi^{(k)}}{\partial x^{2}}+\frac{5 h}{12(1+\mu)} \frac{\partial E^{(k)}}{\partial y}\left(\frac{\partial w^{(k)}}{\partial y}-\varphi_{y}^{(k)}\right)=0 \\
\nabla^{2} \nabla^{2} \psi^{(k)}+\kappa B^{(k)} \frac{\partial^{2} w^{(k)}}{\partial x^{2}}-2 \frac{\mathrm{d} E^{(k)}}{E^{(k)} \mathrm{d} y}\left(\frac{\partial^{3} \psi^{(k)}}{\partial y^{3}}+\frac{\partial^{3} \psi^{(k)}}{\partial x^{2} \partial y}\right)+\frac{\mathrm{d}^{2} E^{(k)}}{E^{(k)} \mathrm{d} y^{2}}\left(\mu \frac{\partial^{2} \psi^{(k)}}{\partial x^{2}}-\frac{\partial^{2} \psi^{(k)}}{\partial y^{2}}\right) \\
+2\left(\frac{\mathrm{d} E^{(k)}}{E^{(k)} \mathrm{d} y}\right)^{2}\left(\frac{\partial^{2} \psi^{(k)}}{\partial y^{2}}-\mu \frac{\partial^{2} \psi^{(k)}}{\partial x^{2}}\right)=0
\end{array}\right.
$$

where $D^{(k)}=\frac{E^{(k)} h^{3}}{12\left(1-\mu^{2}\right)}, C^{(k)}=\frac{5 E^{(k)} h}{12(1+\mu)}, k=1,2$ and $h$ is thickness of the cylindrical shell.

The boundary condition of the crack surface is

$$
\left\{\begin{array}{l}
\frac{\partial \varphi_{y}^{(k)}}{\partial y}+\mu \frac{\partial \varphi_{x}^{(k)}}{\partial x}=0 \\
\frac{\partial \varphi_{x}^{(k)}}{\partial y}+\frac{\partial \varphi_{y}^{(k)}}{\partial x}=0 \\
\frac{\partial w^{(k)}}{\partial y}-\varphi_{y}^{(k)}=0 \quad y=0 \\
\frac{\partial^{2} \psi^{(k)}}{\partial x^{2}}=0 \\
\frac{\partial^{2} \psi^{(k)}}{\partial x \partial y}=0
\end{array}\right.
$$




\section{Higher Order Crack TipFields}

The crack tip stress field would be equipped with the same square root singularity as that of homogeneous materials when the material prosperities of different composite materials at the interfaces are continuous[5]. Therefore, the generalized displacements $\varphi_{r}, \varphi_{\theta}, w$ and generalized stress function $\psi$ can be expressed as follows

$$
\begin{aligned}
& \varphi_{x}^{(k)}=\sum_{n=1}^{\infty} f_{n}^{(k)}(\theta) r^{\frac{n}{2}} \\
& \varphi_{y}^{(k)}=\sum_{n=1}^{\infty} g_{n}^{(k)}(\theta) r^{\frac{n}{2}} \\
& w^{(k)}=\sum_{n=1}^{\infty} j_{n}^{(k)}(\theta) r^{\frac{n}{2}} \\
& \psi^{(k)}=\sum_{n=1}^{\infty} \psi_{n}^{(k)}(\theta) r^{\frac{n+2}{2}}
\end{aligned}
$$

where, $f_{n}^{(k)}(\theta) 、 g_{n}^{(k)}(\theta) 、 j_{n}^{(k)}(\theta)$ are eigen-functions.

Substituting Eq.(5) into Eq.(2) and Eq.(3), the coefficients of $r^{-3 / 2}, r^{-1}, \ldots, r^{n / 2-2}$ are linear independent, the each coefficient term must be zero. The obtained equations are solved and the eigen-function are derived as follows

$$
\begin{aligned}
& \left\{\begin{array}{l}
f_{1}^{(k)}=A_{12}^{(k)} \sin \frac{3 \theta}{2}+A_{11}^{(k)} \cos \frac{3 \theta}{2}+\frac{\mu+9}{\mu+1} A_{12}^{(k)} \sin \frac{\theta}{2}+\frac{3 \mu-5}{\mu+1} A_{11}^{(k)} \cos \frac{\theta}{2} \\
g_{1}^{(k)}=A_{11}^{(k)} \sin \frac{3 \theta}{2}-A_{12}^{(k)} \cos \frac{3 \theta}{2}+\frac{\mu-7}{\mu+1} A_{11}^{(k)} \sin \frac{\theta}{2}+\frac{\mu-7}{\mu+1} A_{12}^{(k)} \cos \frac{\theta}{2} \\
j_{1}^{(k)}=A_{13}^{(k)} \sin \frac{\theta}{2} \\
\psi_{1}^{(k)}=A_{15}^{(k)} \sin \frac{3 \theta}{2}+A_{14}^{(k)} \cos \frac{3 \theta}{2}+A_{15}^{(k)} \sin \frac{\theta}{2}+3 A_{14}^{(k)} \cos \frac{\theta}{2}
\end{array}\right. \\
& \left\{\begin{array}{l}
f_{2}^{(k)}=A_{21}^{(k)} \cos \theta+A_{22}^{(k)} \sin \theta \\
g_{2}^{(k)}=-A_{22}^{(k)} \cos \theta-\mu A_{21}^{(k)} \sin \theta \\
j_{2}^{(k)}=A_{23}^{(k)} \cos \theta \\
\psi_{2}^{(k)}=-A_{24}^{(k)}+A_{24}^{(k)} \cos 2 \theta
\end{array}\right.
\end{aligned}
$$




$$
\begin{aligned}
& f_{3}^{(k)}=A_{31}^{(k)} \cos \frac{3 \theta}{2}+A_{32}^{(k)} \sin \frac{3 \theta}{2}+\frac{3(\mu+1)}{\mu-7} A_{31}^{(k)} \cos \frac{\theta}{2}+\frac{3(\mu+1)}{3 \mu+11} A_{32}^{(k)} \sin \frac{\theta}{2}-\frac{4 N_{m} A_{13}^{(k)}}{3 \mu^{2}+8 \mu-11} . \\
& \sin \frac{\theta}{2}-\delta_{2 k} \beta\left[\frac{1}{4} A_{11}^{(k)} \sin \frac{5 \theta}{2}-\frac{1}{4} A_{12}^{(k)} \cos \frac{5 \theta}{2}-\frac{3 \mu-13}{4(3 \mu+11)} A_{11}^{(k)} \sin \frac{\theta}{2}+\frac{5 \mu-43}{4(\mu-7)} A_{12}^{(k)} \cos \frac{\theta}{2}\right] \\
& g_{3}^{(k)}=\frac{7 \mu-1}{3 \mu+11} A_{32}^{(k)} \cos \frac{3 \theta}{2}+\frac{3 \mu-5}{\mu-7} A_{31}^{(k)} \sin \frac{3 \theta}{2}-\frac{3(\mu+1)}{3 \mu+11} A_{32}^{(k)} \cos \frac{\theta}{2}+\frac{3(\mu+1)}{\mu-7} A_{31}^{(k)} \sin \frac{\theta}{2}+ \\
& \frac{4 N_{m} A_{13}^{(k)}}{8 \mu+3 \mu^{2}-11} \cos \frac{\theta}{2}+\frac{4(3 \mu+1) N_{m} A_{13}^{(k)}}{3\left(8 \mu+3 \mu^{2}-11\right)} \cos \frac{3 \theta}{2}+\left[\frac{A_{12}^{(k)}}{4} \sin \frac{5 \theta}{2}+\frac{3 \mu-17}{3(\mu-7)} A_{12}^{(k)} \sin \frac{3 \theta}{2}+\right. \\
& \left.\frac{3 \mu-13}{4(\mu-7)} A_{12}^{(k)} \sin \frac{\theta}{2}+\frac{A_{11}^{(k)}}{4} \cos \frac{5 \theta}{2}+\frac{9 \mu+13}{3 \mu+11} A_{11}^{(k)} \cos \frac{3 \theta}{2}+\frac{21 \mu+101}{4(3 \mu+11)} A_{11}^{(k)} \cos \frac{\theta}{2}\right] \delta_{2 k} \beta \\
& j_{3}^{(k)}=\frac{2(\mu-1) A_{11}^{(k)}}{\mu+1} \cos \frac{\theta}{2}+\frac{2(\mu+7) A_{11}^{(k)}}{3(\mu+1)} \cos \frac{3 \theta}{2}+\frac{2(\mu-1) A_{12}^{(k)}}{\mu+1} \sin \frac{\theta}{2}+A_{33}^{(k)} \sin \frac{3 \theta}{2}-\frac{A_{13}^{(k)}}{12}\left(\cos \frac{3 \theta}{2}+\right. \\
& \left.3 \cos \frac{\theta}{2}\right) \delta_{2 k} \beta-\frac{\kappa}{C^{(1)}}\left[\frac{A_{15}^{(k)}}{16} \sin \frac{5 \theta}{2}+\frac{A_{15}^{(k)}}{8} \sin \frac{\theta}{2}+\frac{15 A_{14}^{(k)}}{16} \cos \frac{3 \theta}{2}+\frac{15 A_{14}^{(k)}}{8} \cos \frac{\theta}{2}+\frac{3 A_{14}^{(k)}}{16} \cos \frac{5 \theta}{2}\right] \\
& \psi_{3}^{(k)}=A_{35}^{(k)} \sin \frac{5 \theta}{2}+A_{34}^{(k)} \cos \frac{5 \theta}{2}-A_{35}^{(k)} \sin \frac{\theta}{2}-5 A_{34}^{(k)} \cos \frac{\theta}{2}+\frac{\kappa B^{(1)}}{32} A_{13}^{(k)}\left(\sin \frac{\theta}{2}+\right. \\
& \left.\sin \frac{3 \theta}{2}\right)+\frac{\delta_{2 k} \beta}{4}\left(3 A_{14}^{(k)} \sin \frac{3 \theta}{2}+3 A_{14}^{(k)} \sin \frac{\theta}{2}-A_{15}^{(k)} \cos \frac{3 \theta}{2}-3 A_{15}^{(k)} \cos \frac{\theta}{2}\right) \\
& f_{4}^{(k)}=A_{41}^{(k)} \cos 2 \theta+A_{42}^{(k)} \sin 2 \theta-\frac{\mu+1}{\mu-3} A_{41}^{(k)}+\frac{N_{m} A_{23}^{(k)}}{\mu^{2}+2 \mu-3} \\
& \left\{g_{4}^{(k)}=A_{42}^{(k)} \cos 2 \theta-\frac{2 \mu A_{41}^{(k)}}{\mu+3} \sin 2 \theta-\frac{\mu+1}{2} A_{42}^{(k)}-\frac{\mu A_{23}^{(k)} N_{m}}{\mu^{2}+2 \mu-3} \sin 2 \theta\right. \\
& \left\{\begin{array}{l}
j_{4}^{(k)}=A_{43}^{(k)} \cos 2 \theta-\frac{A_{22}^{(k)}}{2} \sin 2 \theta-\frac{\mu-1}{4} A_{21}^{(k)} \\
\psi_{4}^{(k)}=A_{44}^{(k)} \cos 3 \theta+A_{45}^{(k)} \sin 3 \theta-3 A_{45}^{(k)} \sin \theta-A_{44}^{(k)} \cos \theta
\end{array}\right. \\
& \text { where } \delta_{2 k}=\left\{\begin{array}{ll}
0, & k=1, \\
1, & k=2,
\end{array} \text { and } B_{i j}^{(k)}(i=1 \cdots n ; j=1,2,3)\right. \text { are undetermined coefficients. }
\end{aligned}
$$

\section{Continuous Conditions}

Substituting Eq. (6)-(9) into Eq.(5), the generalized displacement fieldsin homogenous material and FGMs region are obtained, and the stress fields will be obtained based on the relationship between the generalized displacement and stress. The stress equations are subject to the following continuous conditions of stress

$$
\sigma_{y}^{(1)}=\sigma_{y}^{(2)}, \tau_{x y}^{(1)}=\tau_{x y}^{(2)}, \tau_{z y}^{(1)}=\tau_{z y}^{(2)} \quad \theta=0
$$

The relations between undetermined coefficients can be obtained as

$$
\begin{aligned}
& A_{11}^{(1)}= A_{11}^{(2)}, \quad A_{12}^{(1)}=A_{12}^{(2)}, \quad A_{13}^{(1)}=A_{13}^{(2)} \\
& A_{14}^{(1)}=A_{14}^{(2)}, \quad A_{15}^{(1)}=A_{15}^{(2)} \\
& A_{21}^{(1)}=A_{21}^{(2)}, \quad A_{22}^{(1)}=A_{22}^{(2)}, \quad A_{23}^{(1)}=A_{23}^{(2)}
\end{aligned}
$$




$$
\begin{gathered}
A_{24}^{(1)}=A_{24}^{(2)} \\
A_{31}^{(1)}=A_{31}^{(2)}-\frac{3 \mu-29}{12(\mu+1)} \beta A_{12}^{(2)} \\
A_{32}^{(1)}=A_{32}^{(2)}+\frac{21 \mu+53}{12(\mu+1)} \beta A_{11}^{(2)} \\
A_{33}^{(1)}=A_{33}^{(2)} \\
A_{34}^{(1)}=A_{34}^{(2)}+\frac{\beta}{4} A_{15}^{(2)} \\
A_{35}^{(1)}=A_{35}^{(2)}+\frac{3 \beta}{4} A_{14}^{(2)} \\
A_{41}^{(1)}=A_{41}^{(2)}, \quad A_{42}^{(1)}=A_{42}^{(2)}, \quad A_{43}^{(1)}=A_{43}^{(2)} \\
A_{44}^{(1)}=A_{44}^{(2)}, \quad A_{45}^{(1)}=A_{45}^{(2)}
\end{gathered}
$$

Substituting Eq.(11)-(21) and Eq.(6)-(9) into Eq.(5), the generalized displacement fields in homogenous material and FGMs regions are obtained finally.

\section{Conclusions}

Based on Reissner plate theory, the higher order fields for an interfacial crack of cylindrical shells are presented. The non-homogeneous material parameter $\beta$ first appeared in the third order field. The curvature only occur in the third order or higher items, and both have evident effect on the higher order items. When $\kappa \rightarrow 0$, the crack-tip higher order fields for FGMs shells can be degenerated into corresponding higher order fields for FGMs plates. The crack-tip fields obtained also have the same property of eigen-function as Williams' solution. They can be applied to the fracture parameter calculation and crack-tip stress analysis in different materials, loads and structures, which is of extensive applicability.

\section{Acknowledgements}

The research is supported by the National Natural Science Foundation of China (No.11172332)

\section{References}

[1] Delale .F. and Erdogan. F.: Journal of Applied Mechanics Vol. 50 (1983), p. 609

[2] Liu Chuntu, Jiang Chiping: Fracture Mechanics for plates and shells (Defense Industry Press,Beijing,2000).

[3] R.A. Arciniega, J.N. Reddy: International Journal of Solids and Structures Vol. 44 (2007), p.2036

[4] F.Delale,F.Erdogan: International Journal of Engineering Science Vol.6 (1988), p.559

[5] Z. H. Jin,N. Noda: Journal of Applied Mechanics Vol.3 (1994), p.738 